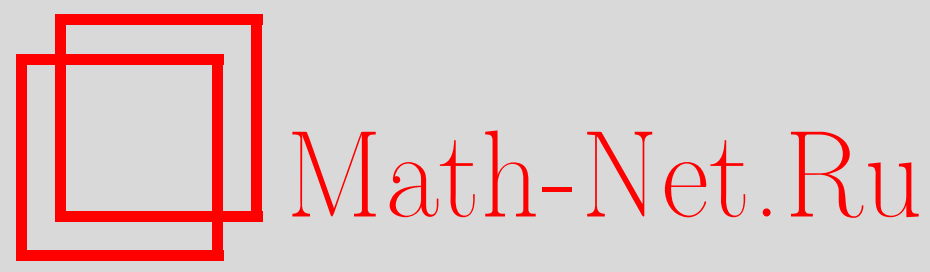

О. А. Старикова, Классы проективно эквивалентных квадрик над локальными кольцами, Дискрет. матем., 2013, том 25, выпуск 2, 91-103

DOI: https://doi.org/10.4213/dm1238

Использование Общероссийского математического портала Math-Net.Ru подразумевает, что вы прочитали и согласны с пользовательским соглашением http://www . mathnet.ru/rus/agreement

Параметры загрузки:

IP: 18.208 .226 .222

26 апреля 2023 г., 13:18:14 


\title{
Классы проективно эквивалентных квадрик над локальными кольцами
}

() 2013 г. О. А. Старикова

\begin{abstract}
Изучаются квадрики и квадратичные формы проективного пространства над локальным кольцом $R=2 R$ с нильпотентным главным максимальным идеалом. Задача перечисления классов проективно эквивалентных квадрик завершена при $\left|R^{*}: R^{* 2}\right|=2$; когда $\left|R^{*}: R^{* 2}\right|=4$, она решена вместе с задачей перечисления классов проективно конгруэнтных квадрик при дополнительном условии.

Работа выполнена при поддержке Российского фонда фундаментальных исследований (код проекта 12-01-00968) и Мин.обр.науки (тема 1.34.11).
\end{abstract}

\section{1. Введение}

В статье завершается решение задачи ([1] - [5] и др.) перечисления классов проективно эквивалентных квадрик и квадратичных форм проективного пространства $R P_{n-1}(n>2)$ над локальным кольцом $R$ с нильпотентным главным максимальным идеалом, когда $R=2 R$ и $\left|R^{*}: R^{* 2}\right|=2$ (Теоремы 2,3 и 4). Тесно связанной является задача классификации схем квадратичных форм полей и колец коэффициентов [3], [6] - [8]. В § 4 мы выявляем число классов проективно конгруэнтных (Теорема 6) и проективно эквивалентных (Теорема 7) квадрик со специальной схемой квадратичных форм порядка 4.

\section{2. Предварительные замечания}

Всюду далее основное кольцо $R$ коэффициентов является ассоциативнокоммутативным и содержит единицу. Через $R^{*}$ обозначаем его мультипликативную группу обратимых элементов.

Проективным пространством, ассоциированным со свободным $R$-модулем $M$, называют (см., например, [9]) множество $P(M)$ всех $R$-свободных прямых слагаемых ранга 1 модуля $M$. Каждое такое слагаемое представляетя в виде $R e$ с элементом $e$ из $M$, на котором подходящая $R$-линейная функция на $M$ принимает значение 1 (унимодулярный элемент).

Пусть $V-$ свободный $R$-модуль ранга $n$. Его векторы будем задавать координатами относительно фиксированного базиса. Унимодулярные векторы над $R$ - это векторы $v=\left(v_{1}, \ldots, v_{n}\right)$, координаты которых порождают единичный идеал кольца $R$, т. е.

$$
\left\langle v_{1}, \ldots, v_{n}\right\rangle=R v_{1}+R v_{2}+\cdots+R v_{n}=R .
$$


Между унимодулярными над $R$ векторами определено отношение

$$
\left(v_{1}, \ldots, v_{n}\right) \sim\left(u_{1}, \ldots, u_{n}\right) \Leftrightarrow \exists t \in R^{*}: v_{i}=t u_{i} \quad \forall i \in\{1, \ldots, n\}
$$

являющееся отношением эквивалентности. Когда $R-$ локальное кольцо, это позволяет определить ассоциированное с $V$ проективное пространство более явно. Классы эквивалентности по отношению " " множества унимодулярных над $R$ векторов есть по определению точки ассоциированного с $V$ проективного пространства $R P_{n-1}$ над кольцом $R$. Далее обозначение $v=\left(v_{1}, \ldots, v_{n}\right)$ используем как для вектора $v \in V$, так и для класса эквивалентности с представителем $v$.

Определение 1. Пусть $M$ и $N$ - свободные модули над коммутативными кольцами $A$ и $B$ соответственно. Биективное отображение $\alpha: P(M) \rightarrow P(N)$ называют проективностъю, если при любых $p_{1}, p_{2}, p_{3} \in P(M)$ имеем $\alpha p_{1} \subset \alpha p_{2}+\alpha p_{3}$ тогда и только тогда, когда $p_{1} \subset p_{2}+p_{3}$.

Для кольцевого гомоморфизма $\sigma: A \rightarrow B$ отображение $\Phi: M \rightarrow N$ называется $\sigma$-полулинейным, если оно аддитивно и

$$
\Phi(a m)=\sigma(a) \Phi(m) \quad(a \in A, m \in M) .
$$

Индуцированное отображение $P(\Phi): P(M) \rightarrow P(N)$ ассоциированных проективных пространств получим, полагая $P(\Phi)(A e)=B \Phi(e)$ для всякого унимодулярного элемента $e$ модуля $M$. Следующая обобщенная основная теорема проективной геометрии доказана в [9].

Теорема 1. Пусть $M$ и $N$ - свободнъе модули конечного ранга не менъше 3 над коммутативными кольцами $A$ и $B$ соответственно. Тогда для любой проективности $\alpha: P(M) \rightarrow P(N)$ существует изоморфизм $\sigma: A \rightarrow B$ и $\sigma$-полулинейный изоморфизм $\Phi: M \rightarrow N$ такие, что $\alpha=P(\Phi)$. Если $\sigma_{i}: A \rightarrow B-$ изоморбизм и $\Phi_{i}: M \rightarrow N$ есть $\sigma_{i}$-полулинейный изоморфизм, $i=1,2$, причем $P\left(\Phi_{1}\right)=P\left(\Phi_{2}\right)$, mо $\sigma_{1}=\sigma_{2}$ и существует maкое $b \in B$, ито $\Phi_{1}=b \Phi_{1}$.

Отметим, что проективности проективного пространства на себя, называемые также его проективными преобразованиями, образуют группу по умножению. Пусть $V$ - свободный $R$-модуль конечного ранга. Проективные линейные $(\sigma$-полулинейные для $\sigma=1)$ преобразования проективного пространства $P(V)$ по умножению образуют группу $R \Gamma(V)$, изоморфную группе $P G L(V)$ - факторгруппе группы $G L(V)$ обратимых линейных преобразований $V$ по ее центру, см. также [10]. Через $R A(V)$ обозначим группу автоморфизмов проективного пространства $P(V)$, которые индуцированы автоморфизмами основного кольца $R$. Ясно, что $R A(V)$ и $R \Gamma(V)$ являются подгруппами группы проективных преобразований проективного пространства $P(V)$, причем $R \Gamma(V)$ есть нормальная подгруппа. Более того, из теоремы 1 , в частном случае, когда $A=B=R$ и $M=N=V$, вытекает следующее утверждение.

Лемма 1. Группа проективностей проективного пространства $P(V)$ на себя совnадает с произведением группь $R \Gamma(V)$ на $R A(V)$.

Квадрикой проективного пространства $R P_{n-1}$, ассоциированного со свободным $R$-модулем ранга $n$, называют проективное многообразие его точек $R^{*} v$, определенное уравнением $v A v^{T}=0$ с ненулевой симметричной $n \times n$-матрицей $A$ над $R$. 
Симметрические $n \times n$-матрицы $A, B$ и соответствующие им квадрики называем проективно конгруэнтными, если существуют такие $k \in R^{*}$ и $U \in G L(n, R)$, что $k A=U B U^{T}$; при $k=1$ матрицы $A$ и $B$ также называем конгруэнтными.

К одной из основных в теории квадрик относят задачу их классификации с точностью до проективностей. Квадрики, переводимые друг в друга проективностью, называют проективно эквивалентными.

Отношения проективной конгруэнтности и проективной эквивалентности квадрик пространств $R P_{n-1}(n>2)$ над некоторыми локальными кольцами главных идеалов (например, при $R=Z_{p^{m}}$ ) совпадают. Поэтому исследуем квадрики вначале с точностью до проективной конгруэнтности.

\section{3. Классы проективно конгруэнтных квадрик}

Задача классификации квадрик тесно связана с исследованием симметричных форм и их матриц. В [1, Следствие 3.4] показано, что над локальным кольцом с обратимым элементом 2 ортогональный базис допускают лишь "сильно невырожденные" симметричные формы. В случае, когда $R=2 R$ есть локальное кольцо с главным максимальным идеалом $J=\langle\varepsilon\rangle$, всякая симметричная матрица $A$ над $R$ конгруэнтна диагональной матрице

$$
\operatorname{diag}\left(k_{1} \varepsilon^{t_{1}}, k_{2} \varepsilon^{t_{2}}, \ldots, k_{r} \varepsilon^{t_{r}}, 0, \ldots, 0\right)
$$

с однозначно определенными показателями $0 \leqslant t_{1} \leqslant t_{2} \leqslant \cdots \leqslant t_{r}, \varepsilon^{t_{r}} \neq 0$ и $k_{i} \in R^{*}$ [2, Теорема 1.3]. Кроме того, если $1+J \subseteq R^{* 2}$ и $\left|R^{*}: R^{* 2}\right|=2$, то известен и нормальный вид. Позднее [11] отмечалось, что включение $1+J \subseteq R^{* 2}$ выполняется, когда $2 \in R^{*}$ и верно по крайней мере одно из утверждений: кольцо $R$ полное, идеал $J$ нильпотентный, кольцо $R$ конечное.

Комбинаторное выражение числа классов проективно конгруэнтных квадрик проективного пространства $R P_{n-1}$ получено в [2] для случая нильпотентного максимального идеала в $R$.

Пусть $\left(\begin{array}{c}p \\ q\end{array}\right)^{\prime}$ равно $\left(\begin{array}{c}p \\ q\end{array}\right)$ для целых чисел $p \geqslant q \geqslant 0$ и равно 0 в других случаях; $\Omega_{q}(m)$ - совокупность упорядоченных наборов $\left(n_{1}, \ldots, n_{q}\right)$ целых чисел $n_{j}>0$ с суммой $m ;[\cdot]$ - целая часть числа.

Теорема 2 ([2], Теорема 3.2). Пусть $R$ - локальное кольцо с нильпотентным степени s главным максимальным идеалом, $2 \in R^{*} u\left|R^{*}: R^{* 2}\right|=2$. Тогда число $N(n, s)$ классов проективно конгруэнтных квадрик пространства $R P_{n-1}(n>2) 8$ случаях $R^{*} \cap\left(1+R^{2}\right) \nsubseteq R^{* 2}$ u $1+R^{* 2} \subseteq R^{* 2}$ равно соответственно

$$
\begin{gathered}
\sum_{m=1}^{n} \sum_{q=1}^{\min \{m, s\}}\left(\begin{array}{c}
s \\
q
\end{array}\right) 2^{q-1}\left\{\left(\begin{array}{c}
m / 2-1 \\
q-1
\end{array}\right)^{\prime}+\left(\begin{array}{c}
m-1 \\
q-1
\end{array}\right)\right\}, \\
\sum_{m=1}^{n} \sum_{q=1}^{\min \{m, s\}}\left(\begin{array}{c}
s \\
q
\end{array}\right)\left\{\left(\begin{array}{c}
m / 2-1 \\
q-1
\end{array}\right)^{\prime}+\sum_{\left(n_{1}, \ldots, n_{q}\right) \in \Omega_{q}(m)}\left[\frac{1}{2} \prod_{j=1}^{q}\left(n_{j}+1\right)\right]\right\} .
\end{gathered}
$$


Применяя методы интегрального представления комбинаторных сумм, Г.П. Егорычев и Е.В. Зима [4] нашли простые формулы для числа $N(n, s)$ из теоремы 2. Теоремы 1 и 2 из [4] резюмирует

Теорема 3. Если $1+R^{* 2} \subseteq R^{* 2}$, то

$$
N(n, s)=\frac{1}{2}\left(\begin{array}{c}
n+2 s \\
2 s
\end{array}\right)+\frac{1}{2}\left(\begin{array}{c}
\lfloor n / 2\rfloor+s \\
s
\end{array}\right)-1 .
$$

Eсли $R^{*} \cap\left(1+R^{2}\right) \nsubseteq R^{* 2} u S(n, s):=\Sigma_{q=0}^{s} 2^{q-1}\left(\begin{array}{c}s \\ q\end{array}\right)\left(\begin{array}{c}n \\ q\end{array}\right), m o$

$$
N(n, s)=S(n, s)+S(\lfloor n / 2\rfloor, s)-1 \text {. }
$$

В [4, стр. 1435] сформулирована также задача о независимом алгебраическом доказательстве и интерпретации формул (2) и (3).

Замечание 1. Отметим, что числа $\left(\begin{array}{c}n+2 s \\ 2 s\end{array}\right)$ и $\left(\begin{array}{c}\lfloor n / 2\rfloor+s \\ s\end{array}\right)$ в формуле (2) (аналогично, $2 S(n, s)$ и $2 S(\lfloor n / 2\rfloor, s)$ в формуле $(3))$ выражают соответственно число $L$ всех классов конгруэнтных $n \times n$-матриц и число $L_{1}$ классов конгруэнтных матриц, инвариантных относительно проективной конгруэнтности. Алгебраическое доказательство теоремы 2.2 приведено в [5], см. также обзор [12].

Нам потребуется следующее утверждение для выявления числа классов проективно эквивалентных квадрик

Следствие 1. Число $L_{2}$ классов конгруэнтных $n \times n$-матрии, неинвариантных относительно проективной конгруэнтности, в случаях $1+R^{* 2} \subseteq R^{* 2} u R^{*} \cap(1+$ $\left.R^{2}\right) \nsubseteq R^{* 2}$ равно соответственно

$$
\begin{gathered}
\left(\begin{array}{c}
n+2 s \\
2 s
\end{array}\right)-\left(\begin{array}{c}
\lfloor n / 2\rfloor+s \\
s
\end{array}\right), \\
\sum_{q=0}^{s} 2^{q}\left(\begin{array}{c}
s \\
q
\end{array}\right)\left(\begin{array}{c}
n \\
q
\end{array}\right)-\sum_{q=0}^{s} 2^{q}\left(\begin{array}{c}
s \\
q
\end{array}\right)\left(\begin{array}{c}
\lfloor n / 2\rfloor \\
q
\end{array}\right) .
\end{gathered}
$$

\section{4. Классы проективно эквивалентных квадрик}

Цель параграфа - перечислить классы проективно эквивалентных квадрик проективного пространства $R P_{n-1}(n>2)$, когда $R$ есть локальное кольцо с нильпотентным ступени $s$ главным максимальным идеалом $J=\langle\varepsilon\rangle,\left|R^{*}: R^{* 2}\right|=2$ и $R=2 R$.

Если элементы $\varepsilon$ и $k \varepsilon$ для фиксированного неквадрата $k$ неавтоморфны в $R$ (например, при $R=Z_{p^{m}}$ ), то отношения проективной конгруэнтности и проективной эквивалентности квадрик пространства $R P_{n-1}(n>2)$ совпадают и число классов проективно эквивалентных квадрик равно $N(n, s)$. Поэтому исследуем случай, когда элемент $\varepsilon$ автоморфен $k \varepsilon$ для фиксированного неквадрата $k$ в $R$.

Как и выше, $N(n, s)$ - число классов проективно конгруэнтных квадрик проективного пространства $R P_{n-1}(n>2), L_{1}$ (соответствено, $\left.L_{2}\right)$ - число классов конгруэнтных матриц, инвариантных (соответственно, неинвариантных) относительно проективной конгруэнтности. 
Число $U(n, m)$ в случае $1+R^{* 2} \subseteq R^{* 2}$ определим равенством

$$
U(n, m)=N(n, s-m)+\sum_{q=1}^{\lfloor n / 2\rfloor}\left(\begin{array}{c}
q+m-1 \\
q
\end{array}\right)(1+N(n-2 q, s-m)),
$$

а при $R^{*} \cap\left(1+R^{2}\right) \nsubseteq R^{* 2}$ полагаем

$$
U(n, m)=N(n, s-m)+\sum_{q=1}^{\lfloor n / 2\rfloor} 2^{q}\left(\begin{array}{c}
m \\
q
\end{array}\right) \sum_{r=q}^{\lfloor n / 2\rfloor}\left(\begin{array}{c}
r-1 \\
q-1
\end{array}\right)(1+N(n-2 r, s-m)) .
$$

Лемма 2. Число классов проективно конгруэнтных квадрик проективного пространства $R P_{n-1}(n>2)$, инвариантных относительно проективной эквивалентности, равно

$$
U(n, s-\lfloor s / 2\rfloor)+U(n,\lfloor s / 2\rfloor)-\left(L_{1}-1\right) .
$$

Доказательство. Пусть $R$ - локальное кольцо с нильпотентным ступени $s$ главным максимальным идеалом, $2 \in R^{*},\left|R^{*}: R^{* 2}\right|=2$. На случай, когда $1+R^{* 2} \subseteq R^{* 2}$ и, следовательно, $R^{*}=R^{* 2} \cup\left(-R^{* 2}\right)$, распространяется закон инерции вещественных квадратичных форм [2, Теорема 2.1]: всякая симметричная $n \times n$-матрица над кольцом $R$ конгруэнтным преобразованием приводится к виду $\left(E_{r}\right.$ - единичная матрица порядка $r$ )

$$
\operatorname{diag}\left(-\varepsilon^{i} E_{s_{i}}, \varepsilon^{i} E_{r_{i}-s_{i}}, \ldots,-\varepsilon^{m} E_{s_{m}}, \varepsilon^{m} E_{r_{m}-s_{m}}, 0, \ldots, 0\right),
$$

где $0 \leqslant i<\ldots<m, \quad 0 \leqslant s_{i} \leqslant r_{i}, \ldots, 0 \leqslant s_{m} \leqslant r_{m}, \quad \varepsilon^{m} \neq 0, \quad r_{i}+\ldots+r_{m} \leqslant n$, причем показатели $i, \cdots, m$ и целые числа $r_{i}, \cdots, r_{m}, s_{i}, \cdots, s_{m}$ не зависят от способа приведения. Поэтому каждая симметричная матрица порядка $n$ определяется однозначно, с точностью до конгруэнтности, произвольным (неупорядоченным) набором $n$ диагональных элементов из множества $\left\{0, \pm 1, \pm \varepsilon, \pm \varepsilon^{2}, \ldots, \pm \varepsilon^{s-1}\right\}$. Найдем число классов проективно конгруэнтных квадрик, инвариантных относительно проективной эквивалентности. Рассмотрим инвариантный относительно проективной эквивалентности класс проективно конгруэнтных квадрик с представителем $A$ вида (4). В зависимости от условий $\varphi(A)$ конгруэнтна $A$ (соответственно, $-A$ ) мы должны иметь для любого четного (соответственно, нечетного) показателя $j(i \leqslant j \leqslant m)$ равенство $r_{j}=2 s_{j}$. Пусть вначале $\varphi(A)$ конгруэнтна $A$. Тогда $A$ характеризуется набором $q$ элементов множества $\left\{1, \varepsilon^{2}, \ldots\right\}$ четных степеней элемента $\varepsilon$ и набором $n-2 q$ элементов множества $\left\{0, \pm \varepsilon, \pm \varepsilon^{3}, \ldots\right\}$. Для четной или нечетной ступени нильпотентности $s$ максимального идеала число таких квадрик равно соответственно

$$
N\left(n, \frac{s}{2}\right)+\sum_{q=1}^{\lfloor n / 2\rfloor}\left(\begin{array}{c}
q+\frac{s}{2}-1 \\
q
\end{array}\right)\left(1+N\left(n-2 q, \frac{s}{2}\right)\right)
$$

или

$$
N\left(n, \frac{s-1}{2}\right)+\sum_{q=1}^{\lfloor n / 2\rfloor}\left(\begin{array}{c}
q+\frac{s+1}{2}-1 \\
q
\end{array}\right)\left(1+N\left(n-2 q, \frac{s-1}{2}\right)\right) .
$$

Если $\varphi(A)$ конгруэнтна $-A$, то $A$ характеризуется набором $q$ элементов множества $\left\{\varepsilon, \varepsilon^{3}, \ldots\right\}$ нечетных степеней элемента $\varepsilon$ и набором $n-2 q$ элементов множества 
$\left\{0, \pm 1, \pm \varepsilon^{2}, \ldots\right\}$. Число таких квадрик для четного или нечетного $s$ равно соответственно

$$
N\left(n, \frac{s}{2}\right)+\sum_{q=1}^{\lfloor n / 2\rfloor}\left(\begin{array}{c}
q+\frac{s}{2}-1 \\
q
\end{array}\right)\left(1+N\left(n-2 q, \frac{s}{2}\right)\right)
$$

или

$$
N\left(n, \frac{s+1}{2}\right)+\sum_{q=1}^{\lfloor n / 2\rfloor}\left(\begin{array}{c}
q+\frac{s-1}{2}-1 \\
q
\end{array}\right)\left(1+N\left(n-2 q, \frac{s+1}{2}\right)\right) .
$$

Пусть теперь матрица $\varphi(A)$ конгруэнтна как матрице $A$, так и матрице $-A$. В этом случае матрицу $A$ характеризует ненулевой набор $\lfloor n / 2\rfloor$ элементов множества $\left\{0, \varepsilon, \varepsilon^{2}, \ldots, \varepsilon^{s-1}\right\}$. Число таких наборов, а следовательно, и классов проективно конгруэнтных квадрик (равное числу классов ненулевых проективно конгруэнтных матриц) с представителями $A$ равно

$$
\left(\begin{array}{c}
\lfloor n / 2\rfloor+s \\
s
\end{array}\right)-1=L_{1}-1 .
$$

Таким образом, число классов проективно конгруэнтных квадрик, инвариантных относительно проективной эквивалентности, равно

$$
U(n, s-\lfloor s / 2\rfloor)+U(n,\lfloor s / 2\rfloor)-\left(L_{1}-1\right) .
$$

При $R^{*} \cap\left(1+R^{2}\right) \nsubseteq R^{* 2}$ и $k \in R^{*} \backslash R^{* 2}$ имеем $R^{*}=R^{* 2} \cup k R^{* 2}$. Согласно [2, Теорема 2.2] всякая симметричная $n \times n$-матрица над $R$ конгруэнтна единственной диагональной матрице вида

$$
\begin{gathered}
\operatorname{diag}(\underbrace{\delta_{i} \varepsilon^{i}, \varepsilon^{i}, \cdots, \varepsilon^{i}}_{r_{i}}, \cdots, \underbrace{\delta_{m} \varepsilon^{m}, \varepsilon^{m}, \cdots, \varepsilon^{m}}_{r_{m}}, 0, \cdots, 0), \\
\delta_{i}, \cdots, \delta_{m} \in\{1, k\}, \quad 0 \leqslant i<\cdots<m, \quad \varepsilon^{m} \neq 0, \quad r_{i}+\ldots+r_{m} \leqslant n .
\end{gathered}
$$

Матрица (5) характеризуется набором коэффициентов $\delta_{i}, \cdots, \delta_{m}$ и кортежем показателей $(i, \ldots, m)$. Найдем число классов проективно конгруэнтных квадрик, инвариантных относительно проективной эквивалентности.

Рассмотрим инвариантный относительно проективной эквивалентности класс проективно конгруэнтных квадрик с представителем $A$ вида (5). В зависимости от условий $\varphi(A)$ конгруэнтна $A$ (соответственно, $\varphi(A)$ конгруэнтна $k A$ ) для любого нечетного (соответственно, четного) показателя $j(i \leqslant j \leqslant m$ ) должно выполняться условие $r_{j} \equiv 0(\bmod 2)$.

Пусть вначале $\varphi(A)$ конгруэнтна $k A$. Обозначим через $q$ количество различных четных показателей $j(i \leqslant j \leqslant m)$ в матрице $(5)$, а через $2 r$ сумму соответствующих четным показателям $j$ чисел $r_{j}$. Тогда число классов проективно конгруэнтных квадрик с матрицами $k A$, конгруэнтными $\varphi(A)$, в случае четной ступени нильпотентности $s$ равно

$$
N\left(n, \frac{s}{2}\right)+\sum_{q=1}^{\lfloor n / 2\rfloor} 2^{q}\left(\begin{array}{c}
\frac{s}{2} \\
q
\end{array}\right) \sum_{r=q}^{\lfloor n / 2\rfloor}\left(\begin{array}{c}
r-1 \\
q-1
\end{array}\right)\left(1+N\left(n-2 r, \frac{s}{2}\right)\right),
$$


а при нечетном $s$ получаем

$$
N\left(n, \frac{s-1}{2}\right)+\sum_{q=1}^{\lfloor n / 2\rfloor} 2^{q}\left(\begin{array}{c}
\frac{s+1}{2} \\
q
\end{array}\right) \sum_{r=q}^{\lfloor n / 2\rfloor}\left(\begin{array}{c}
r-1 \\
q-1
\end{array}\right)\left(1+N\left(n-2 r, \frac{s-1}{2}\right)\right) .
$$

Если $\varphi(A)$ конгруэнтна $A$, то, обозначая через $q$ количество различных нечетных показателей $j(i \leqslant j \leqslant m$ ) в матрице $(5)$, а через $2 r$ сумму соответствующих нечетным показателям $j$ чисел $r_{j}$, находим число классов проективно конгруэнтных квадрик с матрицами $A$ для четной и, соответственно, нечетной ступени нильпотентности $s$ :

$$
N\left(n, \frac{s}{2}\right)+\sum_{q=1}^{\lfloor n / 2\rfloor} 2^{q}\left(\begin{array}{c}
\frac{s}{2} \\
q
\end{array}\right) \sum_{r=q}^{\lfloor n / 2\rfloor}\left(\begin{array}{c}
r-1 \\
q-1
\end{array}\right)\left(1+N\left(n-2 r, \frac{s}{2}\right)\right),
$$

и

$$
N\left(n, \frac{s+1}{2}\right)+\sum_{q=1}^{\lfloor n / 2\rfloor} 2^{q}\left(\begin{array}{c}
\frac{s-1}{2} \\
q
\end{array}\right) \sum_{r=q}^{\lfloor n / 2\rfloor}\left(\begin{array}{c}
r-1 \\
q-1
\end{array}\right)\left(1+N\left(n-2 r, \frac{s+1}{2}\right)\right) .
$$

Пусть теперь матрица $\varphi(A)$ конгруэнтна как матрице $A$, так и матрице $k A$. Число проективно конгруэнтных классов квадрик с такими матрицами находим, исходя из условия четности всех чисел $r_{i}, \ldots, r_{m}$ :

$$
\sum_{q=1}^{s} 2^{q}\left(\begin{array}{c}
s \\
q
\end{array}\right)\left(\begin{array}{c}
\lfloor n / 2\rfloor \\
q
\end{array}\right)=\sum_{q=0}^{s} 2^{q}\left(\begin{array}{c}
s \\
q
\end{array}\right)\left(\begin{array}{c}
\lfloor n / 2\rfloor \\
q
\end{array}\right)-1=L_{1}-1 .
$$

Таким образом, число классов проективно конгруэнтных квадрик, инвариантных относительно проективной эквивалентности, равно

$$
U(n, s-\lfloor s / 2\rfloor)+U(n,\lfloor s / 2\rfloor)-\left(L_{1}-1\right) .
$$

Лемма доказана.

Пусть $U(n, m)$ выбраны как в лемме 2 .

Теорема 4. Число классов проективно эквивалентных квадрик проективного пространства $R P_{n-1}, n>2$, равно

$$
\frac{1}{2}\left(U(n, s-\lfloor s / 2\rfloor)+U(n,\lfloor s / 2\rfloor)+\frac{1}{2} L_{2}\right) .
$$

Доказательство. Пусть $R$ - локальное кольцо с нильпотентным ступени $s$ главным максимальным идеалом, $2 \in R^{*}$ и $\left|R^{*}: R^{* 2}\right|=2$. Найдем число классов проективно эквивалентных квадрик проективного пространства $R P_{n-1}, n>2$. Обозначим через $n_{1}$ (соответственно, $n_{2}$ ) число классов проективно конгруэнтных квадрик инвариантных (неинвариантных) относительно проективной эквивалентности. Пусть $\varphi$ - автоморфизм проективного пространства $R P_{n-1}, n>2$, индуцированный автоморфизмом кольца $R$ и удовлетворяющий условию $\varphi(\varepsilon)=k \varepsilon$. В случае, когда

4 Дискретная математика, т.25 №2 
матрицы $A$ и $\varphi(A)$ проективно конгруэнтны, класс проективно конгруэнтных квадрик с представителем $A$, очевидно, является также классом проективно эквивалентных квадрик. В оставшихся случаях $A$ и $\varphi(A)$ представляют два различных класса проективно конгруэнтных квадрик. Таким образом, число классов проективно эквивалентных квадрик равно

$$
n_{1}+\frac{1}{2} n_{2}=\frac{1}{2}\left(n_{1}+n_{2}\right)+\frac{1}{2} n_{1} .
$$

Число $n_{1}+n_{2}$ есть число $N(n, s)$ всех классов проективно конгруэнтных квадрик, найденное в теореме 2 (см. также теорему 3 ). В силу леммы 2 число классов проективно конгруэнтных квадрик, инвариантных относительно проективной эквивалентности, равно

$$
n_{1}=U(n, s-\lfloor s / 2\rfloor)+U(n,\lfloor s / 2\rfloor)-\left(L_{1}-1\right) .
$$

Но, согласно утверждению теоремы 3,

$$
n_{1}+n_{2}=N(n, s)=L_{1}-1+\frac{1}{2} L_{2}
$$

Поэтому искомое число классов проективно эквивалентных квадрик равно

$$
\frac{1}{2}\left(n_{1}+n_{2}\right)+\frac{1}{2} n_{1}=\frac{1}{2}\left(U(n, s-\lfloor s / 2\rfloor)+U(n,\lfloor s / 2\rfloor)+\frac{1}{2} L_{2}\right) .
$$

Теорема доказана.

При $n=3$ теорема дает число классов проективно эквивалентных квадрик проективной плоскости, выявлявшееся ранее в [13].

Следствие 2. [13, Теорема 5]. Пусть $N$ есть число классов проективно эквивалентных квадрик проективной плоскости $R P_{2}$ над локальным кольцом $R$ с нильпотентным ступени s главным максимальным идеалом $J=\langle\varepsilon\rangle$. Допустим, что $2 \in R^{*},\left|R^{*}: R^{* 2}\right|=2$ и элементы $\varepsilon$ и $k \varepsilon$ в кольце $R$ автоморфны для неквадрата $k$. Тогда для четного или нечетного числа s число $N$ равно, соответственно,

$$
\left(s\left(5 s^{2}+15 s+28\right)\right) / 12 \quad \text { или }\left(5 s^{3}+15 s^{2}+31 s-3\right) / 12,
$$

если $R^{*} \cap\left(1+R^{2}\right) \nsubseteq R^{* 2}$, а при $1+R^{* 2} \subset R^{* 2}$, соответственно,

$$
\left(s\left(5 s^{2}+21 s+28\right)\right) / 12 \quad \text { или } \quad\left(5 s^{3}+21 s^{2}+31 s+3\right) / 12 .
$$

\section{5. Перечисление квадрик и схемы квадратичных форм}

Задача перечисления квадрик и квадратичных форм тесно связана (см. [3], [6] - [8]) с задачей классификации схем квадратичных форм (quadratic form schemes) основных колец коэффициентов, называемых ниже также QF-схемами.

Для элементов $a=r R^{* 2}$ и $b=s R^{* 2}$ группы $G=R^{*} / R^{* 2}$ полагаем 


$$
D(a, b)=\left\{t R^{* 2} \mid t \in\left(r R^{2}+s R^{2}\right) \cap R^{*}\right\} .
$$

Когда $R$ есть поле, известны [8] следующие свойства:

1) $D(1, a)$ - подгруппа группы $G$ и $a \in D(1, a)$;

2) $a \in D(1,-b) \Leftrightarrow b \in D(1,-a)$;

3) $b D(1,-a) \cap D(1,-a c) \cap d D(1,-c) \neq \varnothing \Rightarrow a D(1,-b) \cap D(1,-b d) \cap c D(1,-d) \neq \varnothing$.

Группа $G=R^{*} / R^{* 2}$ с ее отображением $a \mapsto D(1, a)$ и выделенным элементом -1 называется $Q F$-схемой поля $R$. Схема квадратичных форм поля полностью определяет его кольцо Витта [6]-[8].

Схему квадратичных форм определяют также и как абстрактную группу $G=$ $(G, \cdot, 1)$ экспоненты 2 с выделенным элементом $-1 \in G,-a:=(-1) a$, и отображением $a \mapsto D(1, a)$, удовлетворяющим условиям 1) - 3). Эти понятия переносятся и на кольца.

При $\left|R^{*}: R^{* 2}\right|=2$ для локальных колец $R$ существует три QF-схемы $L_{1}, L_{1,1}$ и $L_{1,0}$, определяемые в зависимости от включения $1+R^{* 2} \subseteq R^{* 2}$ или $R^{*} \cap\left(1+R^{2}\right) \nsubseteq R^{* 2}$. Эти QF-схемы соответственно определяются условиями:

$$
\begin{aligned}
& L_{1}: 1 \neq-1, D(1,1)=\{1\} \\
& L_{1,1}: 1 \neq-1, D(1,1)=G \\
& L_{1,0}: 1=-1, D(1,1)=G .
\end{aligned}
$$

$Q F$-схема $L_{1}$, очевидно, реализуется как $Q F$-схема поля, а также локального кольца $R$ с ограничением $1+R^{* 2} \subseteq R^{* 2}$, равносильным равенству $D(1,1)=\{1\}$. Равенство $D(1,1)=G$ эквивалентно условию $R^{*} \cap\left(1+R^{* 2}\right) \nsubseteq R^{* 2}$. Таким образом, для локальных колец с $Q F$-схемами порядка 2 классы квадрик перечисляют Теоремы 2,3 и 4.

Квадратичные схемы порядка 4 могут быть представлены как групповые произведения $L_{1,1} \times L_{1,0}, L_{1,0} \times L_{1,0}, L_{1} \times L_{1,0}$ и групповые расширения $L_{1}[t], L_{1,0}[t], L_{1,1}[t]$ соответствующих $Q F$-схем порядка 2 (см. [8], с.275).

Проиллюстрируем применимость разработанных методов перечисления классов квадрик в случае локального кольца $R$ главных идеалов с индексом $\left|R^{*}: R^{* 2}\right|>2$. Кольца с $Q F$-схемами $L_{1,1} \times L_{1,0}$ и $L_{1,0} \times L_{1,0}$ определяются условием $R^{*} \subseteq R^{2}+b R^{2}$ для всех $b \in R^{*}$, а также $-1 \in R^{*} \backslash R^{* 2}$ или $-1 \in R^{* 2}$ соответственно.

Теорема 5. Пусть $R=2 R$ есть локальное кольцо с главным максимальным идеалом $J=\langle\varepsilon\rangle, 1+J \subseteq R^{* 2},\left|R^{*}: R^{* 2}\right|=4 u R^{*} \subseteq R^{2}+b R^{2}$ для всех $b \in R^{*}$. Тогда

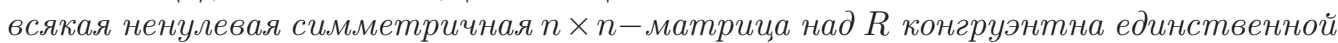
диагональной матрице вида

$$
\begin{gathered}
\operatorname{diag}(\underbrace{\delta_{i} \varepsilon^{i}, \varepsilon^{i}, \cdots, \varepsilon^{i}}_{r_{i}}, \cdots, \underbrace{\delta_{m} \varepsilon^{m}, \varepsilon^{m}, \cdots, \varepsilon^{m}}_{r_{m}}, 0, \cdots, 0), \\
\delta_{i}, \cdots, \delta_{m} \in R^{*} / R^{* 2}, \quad 0 \leqslant i<\cdots<m, \quad \varepsilon^{m} \neq 0, \quad r_{i}+\ldots+r_{m} \leqslant n .
\end{gathered}
$$

Доказательство. Всякая симметричная матрица $A$ над $R$ конгруэнтна диагональной матрице вида (1) [[2], Теорема 1.3]. Диагональная матрица переходит в конгруэнтную матрицу, если какие-либо два ее диагональных элемента вида $a$ и $a b$ умножить на обратимый элемент из $1+b R^{2}$ [[2], Лемма 2.5]. В силу условия $R^{*} \subseteq R^{2}+b R^{2}$ 
для всех $b \in R^{*}$ всякая симметричная матрица $A$ над $R$ конгруэнтна матрице вида (6).

Остается доказать совпадение конгруэнтных матриц $A$ и $D=$ $U A U^{T}$ вида (6). Представим их в клеточно-диагональном виде $\operatorname{diag}\left(f_{1} A_{1}, f_{1} f_{2} A_{2}, \ldots, f_{1} f_{2} \cdots f_{q} A_{q}, O\right), f_{1} f_{2} \cdots f_{q} \neq 0$, где $A_{j}$ - обратимые диагональные клетки и $O$ - нулевая клетка. Тогда при $1 \leqslant t \leqslant q$ клетки $A_{t}$ и $D_{t}$ имеют вид $\operatorname{diag}(\delta, 1, \ldots, 1)$ с $\delta \in R^{*} / R^{* 2}$ и могут различаться лишь значениями $\delta$. Ясно, что для клетки $A_{t}$ значение $\delta=\delta\left(A_{t}\right)$ совпадает с $\left|A_{t}\right|$; аналогично $\delta\left(D_{t}\right)=\left|D_{t}\right|$. По лемме 2.6 из [2] при условии $1+J \subseteq R^{* 2}$ получаем $\left|A_{t}\right| \cdot\left|D_{t}\right| \in R^{* 2}$. Поскольку $\left|A_{t}\right|,\left|D_{t}\right| \in R^{*} / R^{* 2}$, отсюда вытекают равенства $\delta\left(A_{t}\right)=\delta\left(D_{t}\right)$ и $A_{t}=D_{t}$ для всех $t$, и поэтому $A=D$. Теорема доказана.

Теорема 6. Пусть $R$ - локальное кольио с нильпотентным ступени $s$ главным максимальным идеалом, $2 \in R^{*},\left|R^{*}: R^{* 2}\right|=4 u R^{*} \subseteq R^{2}+b R^{2}$ для всех $b \in$ $R^{*}$. Тогда число $\widehat{N}(n, s)$ классов проективно конгруэнтных квадрик пространства $R P_{n-1}(n>2)$ равно

$$
\sum_{q=1}^{s} 4^{q-1}\left(\left(\begin{array}{l}
n \\
q
\end{array}\right)+3\left(\begin{array}{c}
\lfloor n / 2\rfloor \\
q
\end{array}\right)\right)\left(\begin{array}{l}
s \\
q
\end{array}\right) .
$$

Доказательство. Рассмотрим классы конгруэнтных симметрических $n \times n$-матриц над кольцом $R$ с точностью до отношения проективной конгруэнтности. Обозначим через $K_{1}$ (аналогично, $K_{2}$ ) число классов ненулевых конгруэнтных матриц, инвариантных (соответственно, неинвариантных) относительно проективной конгруэнтности. В случае, когда матрицы $k A$ попарно конгруэнтны для $k \in R^{*} / R^{* 2}$, класс конгруэнтных матриц с представителем $A$, очевидно, является также классом проективно конгруэнтных матриц. В оставшихся случаях матрицы $k A$ представляют четыре различных класса конгруэнтных матриц. Таким образом, получаем число классов проективно конгруэнтных квадрик

$$
\widehat{N}(n, s)=K_{1}+\frac{1}{4} K_{2}=\frac{1}{4}\left(\left(K_{1}+K_{2}\right)+3 K_{1}\right)=\frac{1}{4}\left(K+3 K_{1}\right) .
$$

Включение $1+J \subseteq R^{* 2}$ вытекает из условия нильпотентности максимального идеала $J$. Тем самым, для числа $K=K_{1}+K_{2}$ всех классов ненулевых конгруэнтных матриц вида (6) получаем

$$
K=\sum_{q=1}^{s} 4^{q}\left(\begin{array}{l}
s \\
q
\end{array}\right)\left(\begin{array}{l}
n \\
q
\end{array}\right)
$$

Необходимым и достаточным условием конгруэнтности матриц $k_{1} A$ и $k_{2} A$ для $k_{1}, k_{2} \in R^{*} / R^{* 2}$ является четность чисел $r_{i}, \ldots, r_{m}$, поэтому

$$
K_{1}=\sum_{q=1}^{s} 4^{q}\left(\begin{array}{c}
s \\
q
\end{array}\right)\left(\begin{array}{c}
\lfloor n / 2\rfloor \\
q
\end{array}\right)
$$

Резюмируя, получаем утверждение теоремы.

Если элементы $\varepsilon$ и $k \varepsilon$ для $k \in R^{*} / R^{* 2}$ неавтоморфны в $R$, то отношения проективной конгруэнтности и проективной эквивалентности квадрик пространства $R P_{n-1}$ 
совпадают. Остается рассмотреть случай, когда для $k \in R^{*} / R^{* 2}$ существует автоморфизм $\phi_{k}$ кольца $R$, при котором $\phi_{k}(\varepsilon)=k \varepsilon$.

Пусть $R$ - локальное кольцо с нильпотентным ступени $s$ главным максимальным идеалом, $2 \in R^{*},\left|R^{*}: R^{* 2}\right|=4, R^{*} \subseteq R^{2}+b R^{2}$ для всех $b \in R^{*}$ и

$$
V(n, m)=\widehat{N}(n, s-m)+\sum_{q=1}^{\lfloor n / 2\rfloor} 4^{q}\left(\begin{array}{c}
m \\
q
\end{array}\right) \sum_{r=q}^{\lfloor n / 2\rfloor}\left(\begin{array}{c}
r-1 \\
q-1
\end{array}\right)(1+\widehat{N}(n-2 r, s-m)),
$$

где $\widehat{N}(n, s)$ - число классов проективно конгруэнтных квадрик. Числа $K$ всех классов конгруэнтных квадрик и $K_{1}$ классов конгруэнтных квадрик, инвариантных относительно проективной конгруэнтности, найдены в теореме 6.

Теорема 7. В случае, когда элементы $\varepsilon$ и $k \varepsilon$ автоморфны для любого обратимого элемента $k$ из $R$, число классов проективно эквивалентных квадрик пространства $R P_{n-1}(n>2)$ равно

$$
\frac{1}{16}\left(K+12(V(n, s-\lfloor s / 2\rfloor)+V(n,\lfloor s / 2\rfloor))-9 K_{1}\right) .
$$

Доказательство. Найдем число классов проективно эквивалентных квадрик проективного пространства $R P_{n-1}, n>2$. Обозначим через $n_{1}$ (соответственно, $\left.n_{2}\right)$ число классов проективно конгруэнтных квадрик инвариантных (неинвариантных) относительно проективной эквивалентности. Пусть $\phi_{k}-$ автоморфизм проективного пространства $R P_{n-1}$, индуцированный автоморфизмом кольца $R$ и удовлетворяющий условию $\phi_{k}(\varepsilon)=k \varepsilon$. В случае, когда матрицы $A$ и $\phi_{k}(A)$ проективно конгруэнтны, класс проективно конгруэнтных квадрик с представителем $A$, очевидно, является также классом проективно эквивалентных квадрик. В оставшихся случаях $A$ и $\phi_{k}(A)$ представляют четыре различных класса проективно конгруэнтных квадрик. Таким образом, число классов проективно эквивалентных квадрик равно

$$
n_{1}+\frac{1}{4} n_{2}=\frac{1}{4}\left(\left(n_{1}+n_{2}\right)+3 n_{1}\right) .
$$

Число $n_{1}+n_{2}$ есть число $\widehat{N}(n, s)$ всех классов проективно конгруэнтных квадрик, выявленное в теореме 6 .

Найдем число классов проективно конгруэнтных квадрик, инвариантных относительно проективной эквивалентности. Рассмотрим инвариантный относительно проективной эквивалентности класс проективно конгруэнтных квадрик с представителем $A$ вида (6). В зависимости от условий $\phi_{k}(A)$ конгруэнтна $A$ (соответственно, $\phi_{k}(A)$ конгруэнтна $k A$ ) для любого нечетного (соответственно, четного) показателя $j(i \leqslant j \leqslant m)$ должно выполняться условие $r_{j} \equiv 0(\bmod 2)$. Число классов проективно конгруэнтных квадрик с матрицами $k A$, конгруэнтными $\phi_{k}(A)$, в случае четной ступени нильпотентности $s$ равно

$$
\widehat{N}\left(n, \frac{s}{2}\right)+\sum_{q=1}^{\lfloor n / 2\rfloor} 4^{q}\left(\begin{array}{c}
\frac{s}{2} \\
q
\end{array}\right) \sum_{r=q}^{\lfloor n / 2\rfloor}\left(\begin{array}{c}
r-1 \\
q-1
\end{array}\right)\left(1+\widehat{N}\left(n-2 r, \frac{s}{2}\right)\right),
$$

а при нечетном $s$ получаем

$$
\widehat{N}\left(n, \frac{s-1}{2}\right)+\sum_{q=1}^{\lfloor n / 2\rfloor} 4^{q}\left(\begin{array}{c}
\frac{s+1}{2} \\
q
\end{array}\right) \sum_{r=q}^{\lfloor n / 2\rfloor}\left(\begin{array}{c}
r-1 \\
q-1
\end{array}\right)\left(1+\widehat{N}\left(n-2 r, \frac{s-1}{2}\right)\right) .
$$


Аналогично, число классов проективно конгруэнтных квадрик с матрицами $A$, конгруэнтными $\phi_{k}(A)$, для четной и, соответственно, нечетной ступени нильпотентности $s$ равно

$$
\widehat{N}\left(n, \frac{s}{2}\right)+\sum_{q=1}^{\lfloor n / 2\rfloor} 4^{q}\left(\begin{array}{c}
\frac{s}{2} \\
q
\end{array}\right) \sum_{r=q}^{\lfloor n / 2\rfloor}\left(\begin{array}{c}
r-1 \\
q-1
\end{array}\right)\left(1+\widehat{N}\left(n-2 r, \frac{s}{2}\right)\right),
$$

и

$$
\widehat{N}\left(n, \frac{s+1}{2}\right)+\sum_{q=1}^{\lfloor n / 2\rfloor} 4^{q}\left(\begin{array}{c}
\frac{s-1}{2} \\
q
\end{array}\right) \sum_{r=q}^{\lfloor n / 2\rfloor}\left(\begin{array}{c}
r-1 \\
q-1
\end{array}\right)\left(1+\widehat{N}\left(n-2 r, \frac{s+1}{2}\right)\right) .
$$

Пусть теперь матрица $\phi_{k}(A)$ конгруэнтна как матрице $A$, так и матрице $k A$. Число проективно конгруэнтных классов квадрик с такими матрицами находим, исходя из условия четности всех чисел $r_{i}, \ldots, r_{m}$ :

$$
\sum_{q=1}^{s} 4^{q}\left(\begin{array}{c}
s \\
q
\end{array}\right)\left(\begin{array}{c}
\lfloor n / 2\rfloor \\
q
\end{array}\right)=K_{1}
$$

Таким образом, число классов проективно конгруэнтных квадрик, инвариантных относительно проективной эквивалентности, равно

$$
V(n, s-\lfloor s / 2\rfloor)+V(n,\lfloor s / 2\rfloor)-K_{1} .
$$

Но, согласно доказательству теоремы 6 ,

$$
\widehat{N}(n, s)=\frac{1}{4}\left(K+3 K_{1}\right) .
$$

Значит, искомое число классов проективно эквивалентных квадрик равно

$$
\begin{gathered}
\frac{1}{4}\left(\widehat{N}(n, s)+3 n_{1}\right)=\frac{1}{16}\left(K+3 K_{1}+12\left(V(n, s-\lfloor s / 2\rfloor)+V(n,\lfloor s / 2\rfloor)-K_{1}\right)\right)= \\
\frac{1}{16}\left(K+12(V(n, s-\lfloor s / 2\rfloor)+V(n,\lfloor s / 2\rfloor))-9 K_{1}\right) .
\end{gathered}
$$

Теорема доказана.

\section{Список литературы}

1. Милнор Дж., Хьюзмоллер Д., Симметрические билинейные формы. Наука, Москва, 1986.

2. Левчук В. М., Старикова О. А., Квадратичные формы проективных пространств над кольцами. Матем. сборник (2006) 197, №6, 97-110.

3. Левчук В. М., Старикова О. А., Нормальный вид и схемы квадратичных форм. Фундамент. и прикл. матем. (2007) 13, №1, 161-178.

4. Egorychev G. P., Zima E. V., Simple formulae for the number of quadrics and symmetric forms of modules over local rings. Comm. in Algebra (2008) 36, 1426-1436.

5. Старикова О. А., Свистунова А. В., Перечисление квадрик проективных пространств над локальными кольцами. Изв. вузов. Матем. (2011) 12, 59-63. 
6. Kula M., Fields and quadratic form schemes. Ann. Math. Sil. (1985) 1, №13, 7-22.

7. Kula M., Counting Witt rings.. J. Algebra (1998) 206, №2, 568-587.

8. Marshall M., The elementary type conjecture in quadratic form theoryContemp. Math (2004) 344, 275-293.

9. Ojanguren M., Sridharan R., A note on the fundamental theorem of projective geometry. Comm. Math. Helv. (1969) 3, 310-315.

10. О’Мира О., Лекции о линейных группах. В сб. перев. "Автоморфизмы классических гpynn" (1976) 57-167.

11. Cao Yonglin, Szechtman F., Congruence of symmetric matrices over local rings. Linear Algebra Appl. (2009) 431, №9, 1687-1690.

12. Старикова О. А., Квадратичные формы и квадрики пространств над локальными кольцами. Фундамент. и прикл. матем. (2012) 17, №3, 97-110.

13. Старикова О. А., Перечисление квадрик проективных плоскостей и пространств над локальными кольцами главных идеалов. Алгебра и теория моделей (2003) 4, 110-115.

Статья поступила 12.02.2013. 